\title{
Expression of ENT1 Protein in Elderly Patients with Schizophrenia
}

\author{
Dan Shan ${ }^{1}$, Vahram Haroutunian ${ }^{2}$, James H. Meador-Woodruff ${ }^{1,3}$, and Robert E. \\ McCullumsmith ${ }^{1}$ \\ ${ }^{1}$ Department of Psychiatry and Behavioral Neurobiology, University of Alabama at Birmingham, \\ Birmingham, Alabama \\ ${ }^{2}$ Department of Psychiatry, Mount Sinai School of Medicine, New York \\ ${ }^{3}$ Evelyn F. McKnight Brain Institute, University of Arizona, Arizona, USA
}

\begin{abstract}
Alterations in glutamatergic neurotransmission are thought to be involved in several psychiatric disorders, including schizophrenia. Equlibrative nucleoside transporter 1 (ENT1) regulates glutamate levels by regulating excitatory amino acid transporter expression and activity in the brain. In this study, we investigated whether ENT1 is abnormally expressed in the brain of elderly patients with schizophrenia. We measured protein expression of ENT1 in the superior temporal gyrus (STG) and anterior cingulate cortex (ACC) in patients with schizophrenia (STG, $\mathrm{n}=22$; ACC, $n=34$ ) and a comparison group ( $\mathrm{STG}, \mathrm{n}=24$; ACC, $\mathrm{n}=29$ ). We found decreased ENT1 expression in the superior temporal gyrus in patients with schizophrenia, supporting the hypothesis of altered glutamate transport in this illness.
\end{abstract}

\section{Keywords}

Equlibrative nucleoside transporter 1 (ENT1); schizophrenia; glutamate; excitatory amino acid transporter (EAAT); adenosine

\section{Introduction}

We have previously found decreased expression of glutamate transporters in the brain in schizophrenia and we hypothesize that abnormalities in glutamate reuptake contribute to the pathophysiology of this illness [1]. One novel mechanism for the regulation of glutamate transporter expression and activity involves the equilibrative nucleoside transporter type 1 (ENT1). ENT1 facilitates transport of nucleosides, such as adenosine, across biological membranes [2-4]. ENT1 transports adenosine until the concentration of adenosine is equalized on each side of the membrane. Inhibition of ENT transport activity leads to elevated levels of adenosine and increased activation of presynaptic adenosine A1 receptors [5]. However, recent evidence also suggests a role for ENT1 in modulating glutamate levels. For example, inhibition or knockdown of ENT1 diminishes the mRNA expression and glutamate transport activity of excitatory amino acid transporter 2 (EAAT2) [6]. Given this putative role for ENT1, we hypothesize that alterations of ENT1 expression may contribute to glutamatergic abnormalities in patients with schizophrenia. Thus, we examined

\footnotetext{
Address all correspondence to: Robert E. McCullumsmith, M.D., Ph.D., Department of Psychiatry and Behavioral Neurobiology, University of Alabama at Birmingham, School of Medicine, Civitan International Research Center, Room576A, $15303^{\text {rd }}$ Avenue South, Birmingham, AL 35294, Phone: (205) 996-6285, Fax: (205) 975-4879, smithrob@uab.edu.
} 
expression of ENT1 protein in the superior temporal gyrus and anterior cingulate cortex in subjects with schizophrenia and a comparison group.

\section{Materials and Methods}

\section{Subjects and Tissue Preparation}

Subjects from the Mount Sinai Medical Center brain bank were recruited prospectively and underwent a thorough antemortem diagnostic and clinical assessment (Table 1). Subjects were excluded for a history of alcoholism, death by suicide, or coma for more than $6 \mathrm{hrs}$ before death. Next of kin consent was obtained for each subject. For western blot studies, superior temporal gyrus (STG) and anterior cingulate cortex (ACC) were dissected, pulverized in liquid nitrogen, and stored at $-80^{\circ} \mathrm{C}$. For immunofluorescence studies, prefrontal cortex was cryostat sectioned $(15 \mu \mathrm{m})$ and mounted onto microscope slides which were stored at $-80^{\circ} \mathrm{C}$.

\section{Western Blot Analysis}

Western blot samples were prepared and analyzed as previously described [7]. Homogenates were stored at $-80^{\circ} \mathrm{C}$ until analysis.

ENT1 (1:600) and Valosin containing protein (VCP, 1:5000) antibodies were obtained from Abcam (Cambridge, Massachusetts, USA). Blots were imaged with the LiCor Odyssey laser based imaging system.

\section{Immunofluorescence}

Frozen sections were fixed with fresh $4 \%$ formaldehyde in PBS for 10 min at $4{ }^{\circ} \mathrm{C}$, then blocked with 10\% Normal Donkey Serum (Jackson Immuno Research, West Grove, Pennsylvania, USA) for $2 \mathrm{hrs}$ at room temperature. Sections were then incubated in citrate buffer $(10 \mathrm{mM}, \mathrm{pH} 4.0)$ for $10 \mathrm{~min}$ at $37^{\circ} \mathrm{C}$ followed by $20 \mathrm{~min}$ at room temperature in a humidified chamber. Next, sections were incubated with the primary antibodies anti-ENT1 (1:500, Abcam, Cambridge, Massachusetts, USA) and anti-NeuN (1:500, Abcam, Cambridge, Massachusetts, USA) for $16 \mathrm{hrs}$ at $4^{\circ} \mathrm{C}$. Rabbit IgG (Santa Cruz, California, USA) or Mouse $\operatorname{IgG}_{1}$ (Santa Cruz, California, USA) were used as negative controls. After incubation with Donkey anti-Rabbit conjugated with DyLight 488 (1:500, Jackson Immuno Research, West Grove, Pennsylvania, USA) or Donkey anti-Mouse conjugated with DyLight 459 (1:500, Jackson Immuno Research, West Grove, Pennsylvania, USA) for 2 hrs, the sections were examined using a Leica confocal microscope (Leica DM6000 B, Mannheim, Germany; Leica AF 6000 LX, Mannheim, Germany).

\section{Data Analysis}

Using Odyssey 3.0 analytical software, near-infrared emissions detected by the LiCor Odyssey scanner were expressed as integrated intensity with top-bottom median intra-lane background subtraction. In each subject, duplicate lanes of ENT1 protein expression were normalized to VCP as an in-lane loading control and then averaged for each subject. VCP was chosen as a loading control because VCP was previously determined to be unchanged in schizophrenia compared to control subjects [8].

Data were analyzed using Statistica (Statsoft, Tulsa, Oklahoma, USA). Correlation analyses were performed to probe for associations between the expression of ENT1 and tissue $\mathrm{pH}$, age, and postmortem interval. One-way analysis of variance was used when significant correlations were not found. Analysis of variance was performed to assess the effects of antipsychotic medication exposure in subjects with schizophrenia. 


\section{Results}

To determine the localization of ENT1 in human brain, we performed immunofluorescence in sections from the prefrontal cortex (Fig. 1A). ENT1 was expressed in large and small cells throughout the cortical gray matter. ENT1 expression colocalized with the neuronal marker NeuN in cells with large (pyramidal cell-like) and small (interneuron-like) profiles. ENT1 was also expressed in some small cells that were not stained with NeuN. We did not detect a fluorescent signal in sections stained with control antibodies (Data not shown).

We found a significant decrease of ENT1 protein in the superior temporal gyrus $[F(1,46)=$ 7.52, $\mathrm{P}<0.01$ ], but not anterior cingulate cortex, in patients with schizophrenia (Fig. 1B). No significant correlation was found between ENT1 expression and tissue $\mathrm{pH}$, postmortem interval or age. VCP protein expression in schizophrenia subjects was not changed compared to comparison subjects. We did not find differences in ENT1 expression in patients with schizophrenia on antipsychotic medications at the time of death, compared to patients off medications for at least 6 weeks (data not shown).

\section{Discussion}

In this study, we demonstrated that ENT1 protein is expressed in neurons in the frontal cortex, consistent with reports that ENT1 is ubiquitously distributed in human tissue and expressed in neurons and astroglia in cortical regions [5, 9]. We also found that ENT1 protein expression was decreased in the superior temporal gyrus in patients with schizophrenia. These findings support the hypothesis that nucleoside-mediated neurotransmission may be abnormal in patients with schizophrenia, and suggest a mechanism for diminished glutamate reuptake capacity in this illness.

We speculate that diminished ENT1expression could indirectly affect glutamate reuptake via changes in adenosine levels. Decreased ENT1 expression leads to accumulation of extracellular adenosine, which activates synaptic adenosine A1 receptors, diminishing presynaptic glutamate release $[9,10]$. Decreased synaptic glutamate levels may lead to remodeling of synapses including a compensatory decrease in the expression of glutamate transporters such as EAAT2 [11].

Alternatively, diminished ENT1 expression could directly affect glutamate reuptake in patients with schizophrenia. The glutamate transporter EAAT2 is localized to perisynaptic regions in astrocytic processes and linked to mitochondrial membranes through proteinprotein interactions by several scaffolding proteins [12]. ENT1 is expressed in plasma and mitochondrial membranes, and may help stabilize the scaffolding protein complex that anchors EAAT2 in the plasma membrane. A decrease in ENT1 expression could disrupt this complex, leading to increased turnover and degradation of EAAT2 protein.

Regardless of the mechanism, decreased expression of ENT1 may contribute to the pathophysiology of schizophrenia. Changes in the levels of adenosine and/or glutamate in the synaptic cleft can affect NMDA-subtype glutamate receptor- mediated neuroplastic responses [10,13]. Modulation of NMDA receptor function is a well-established pathway linked to symptoms in patients with schizophrenia, as NMDA receptor antagonists such as phencyclidine can cause schizophrenia-like symptoms in otherwise healthy subjects [14, $15]$.

We found decreased ENT protein expression in the superior temporal gyrus, but not the anterior cingulate cortex. Regionally divergent changes in gene expression are not without precedent; Several other studies have found an changes in various gene expression products in one area, but not in another, suggesting that changes in the brain in schizophrenia are not 
global, but may be region specific [16-18]. Given that we only found modest changes in ENT1 expression in one brain region, our findings need to be carefully replicated in another schizophrenia sample.

Previous studies have suggested that antipsychotic medications might alter neurotransmitter transport $[19,20]$. We did not detect any differences in ENT1 protein expression between medicated patients and patients who had been free of antipsychotic medications for 6 weeks prior to death, suggesting our findings are not due to a medication effect.

\section{Conclusion}

We found decreased expression of ENT1 protein in the superior temporal gyrus in patients with schizophrenia. Taken together with our previous findings of altered glutamate transporter expression in this illness, these data support the hypothesis that diminished glutamate reuptake contributes to the pathophysiology of schizophrenia.

\section{Acknowledgments}

We thank Stephen Moore for his assistance with immunofluorescence imaging. This study was supported by MH53327 (JMW), MH88752 (JMW), MH0740106 (REM), Doris Duke Clinical Scientist Award (REM), and MH094445 (REM).

\section{References}

1. Huerta I, et al. Expression of excitatory amino acid transporter interacting protein transcripts in the thalamus in schizophrenia. Synapse. 2006; 59(7):394-402. [PubMed: 16485262]

2. Chen $\mathrm{J}$, et al. The type 1 equilibrative nucleoside transporter regulates anxiety-like behavior in mice. Genes Brain Behav. 2007; 6(8):776-83. [PubMed: 17376149]

3. Governo RJ, et al. Localization of the NBMPR-sensitive equilibrative nucleoside transporter, ENT1, in the rat dorsal root ganglion and lumbar spinal cord. Brain Res. 2005; 1059(2):129-38. [PubMed: 16226730]

4. Goff DC, Coyle JT. The emerging role of glutamate in the pathophysiology and treatment of schizophrenia. Am J Psychiatry. 2001; 158(9):1367-77. [PubMed: 11532718]

5. Baldwin SA, et al. The equilibrative nucleoside transporter family, SLC29. Pflugers Arch. 2004; 447(5):735-43. [PubMed: 12838422]

6 . Wu J, et al. ENT1 regulates ethanol-sensitive EAAT2 expression and function in astrocytes. Alcohol Clin Exp Res. 2010; 34(6):1110-7. [PubMed: 20374202]

7. Hammond JC, et al. Evidence for abnormal forward trafficking of AMPA receptors in frontal cortex of elderly patients with schizophrenia. Neuropsychopharmacology. 2010; 35(10):2110-9. [PubMed: 20571483]

8. Bauer DE, et al. Expression of four housekeeping proteins in elderly patients with schizophrenia. $\mathrm{J}$ Neural Transm. 2009; 116(4):487-91. [PubMed: 19139805]

9. Jennings LL, et al. Distinct regional distribution of human equilibrative nucleoside transporter proteins 1 and 2 (hENT1 and hENT2) in the central nervous system. Neuropharmacology. 2001; 40(5):722-31. [PubMed: 11311901]

10. Benarroch EE. Adenosine and its receptors: multiple modulatory functions and potential therapeutic targets for neurologic disease. Neurology. 2008; 70(3):231-6. [PubMed: 18195269]

11. Wilson NR, et al. Presynaptic regulation of quantal size by the vesicular glutamate transporter VGLUT1. J Neurosci. 2005; 25(26):6221-34. [PubMed: 15987952]

12. Bauer, D., et al. Interactions between GLAST, the Na+/K+ ATPase, and mitochondria: Potential implications for astroglial glutamate metabolism. Society for Neuroscience Annual meeting; 2011; Washington, DC: Society for Neuroscience; 2011.

13. McCullumsmith RE, Clinton SM, Meador-Woodruff JH. Schizophrenia as a disorder of neuroplasticity. Int Rev Neurobiol. 2004; 59:19-45. [PubMed: 15006483] 
14. Smith RE, et al. Expression of excitatory amino acid transporter transcripts in the thalamus of subjects with schizophrenia. Am J Psychiatry. 2001; 158(9):1393-9. [PubMed: 11532723]

15. Clinton SM, Meador-Woodruff JH. Abnormalities of the NMDA Receptor and Associated Intracellular Molecules in the Thalamus in Schizophrenia and Bipolar Disorder. Neuropsychopharmacology. 2004; 29(7):1353-62. [PubMed: 15054476]

16. Katsel $\mathrm{P}$, et al. Variations in differential gene expression patterns across multiple brain regions in schizophrenia. Schizophr Res. 2005; 77(2-3):241-52. [PubMed: 15923110]

17. Kristiansen LV, et al. Expression of the NR2B-NMDA receptor trafficking complex in prefrontal cortex from a group of elderly patients with schizophrenia. Schizophr Res. 2010; 119(1-3):198209. [PubMed: 20347576]

18. Oni-Orisan A, et al. Altered vesicular glutamate transporter expression in the anterior cingulate cortex in schizophrenia. Biol Psychiatry. 2008; 63(8):766-75. [PubMed: 18155679]

19. De Souza IE, McBean GJ, Meredith GE. Chronic haloperidol treatment impairs glutamate transport in the rat striatum. Eur J Pharmacol. 1999; 382(2):139-42. [PubMed: 10528149]

20. Melone M, et al. The expression of glutamate transporter GLT-1 in the rat cerebral cortex is downregulated by the antipsychotic drug clozapine. Mol Psychiatry. 2001; 6(4):380-6. [PubMed: 11443521] 


\section{A}
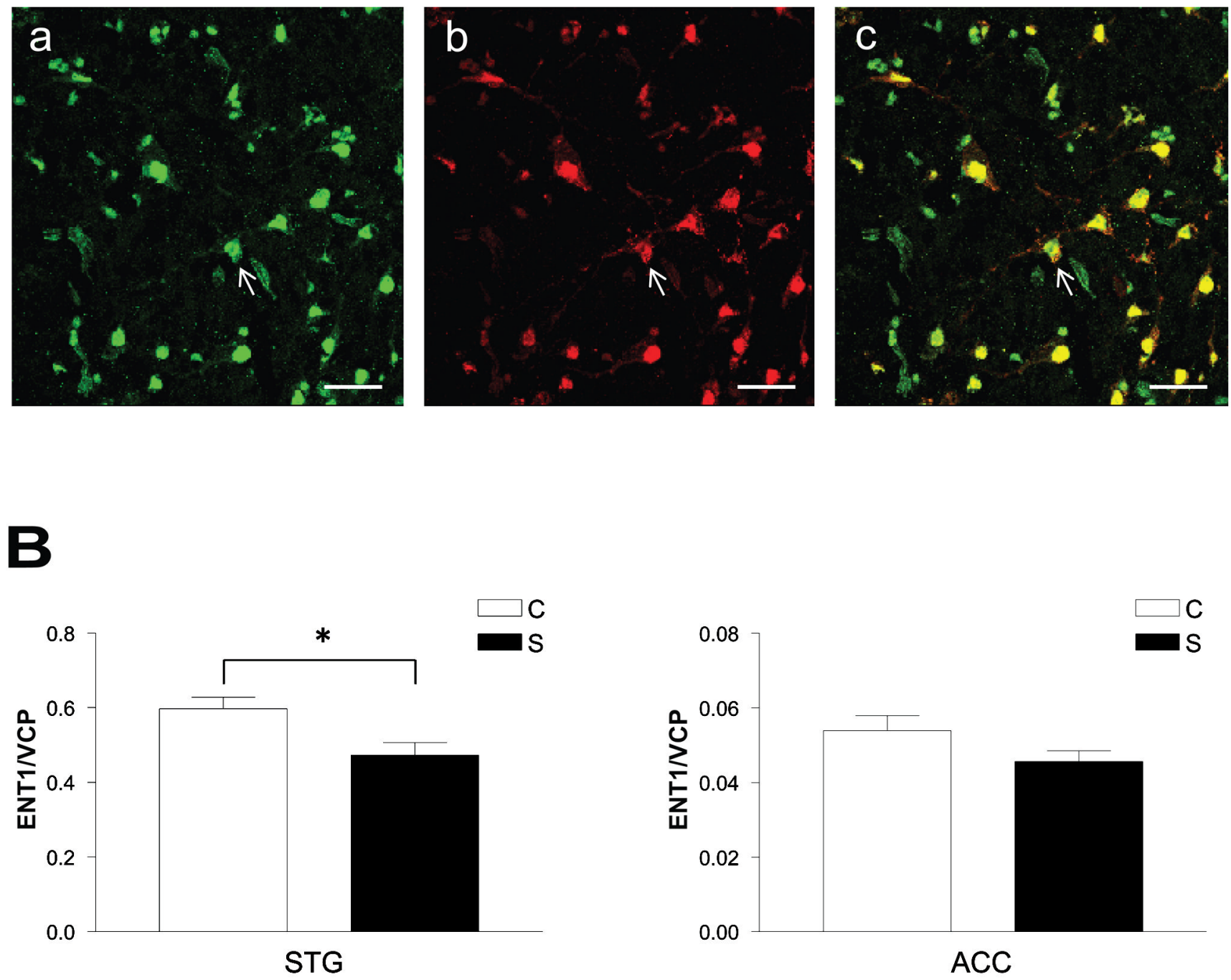

Figure 1.

Figure 1A. ENT1 protein localization in the human frontal cortex. Labeling of cell profiles with (a) NeuN antibody (green), (b) ENT1 antibody (red), (c) merge of NeuN and ENT1 (yellow).

Figure 1B. ENT1 protein expression in the superior temporal gyrus (STG) and anterior cingulate cortex (ACC). Data are presented as a ratio of ENT1 to VCP. Data are expressed as mean \pm standard error. ENT1 was significantly decreased $(\mathrm{P}<0.01)$ in the superior temporal gyrus in schizophrenia. No change was found in the anterior cingulate cortex. comparison group (C); schizophrenia (S). $* \mathrm{P}<0.01$. 
Table 1

Subjects' characteristics

\begin{tabular}{lcccc}
\hline \multicolumn{2}{c}{ STG } & \multicolumn{2}{c}{ ACC } \\
\hline & Comparison & Schizophrenia & Comparison & Schizophrenia \\
$\mathrm{N}$ & 24 & 22 & 29 & 34 \\
$\mathrm{Sex}$ & $14 \mathrm{~m} / 10 \mathrm{f}$ & $16 \mathrm{~m} / 6 \mathrm{f}$ & $12 \mathrm{~m} / 17 \mathrm{f}$ & $22 \mathrm{~m} / 12 \mathrm{f}$ \\
Age (years) & $78 \pm 12.9$ & $73 \pm 11.6$ & $78 \pm 14.5$ & $73 \pm 11.2$ \\
Tissue pH & $6.5 \pm 0.3$ & $6.5 \pm 0.3$ & $6.4 \pm 0.2$ & $6.3 \pm 0.3$ \\
PMI (hours) & $7.9 \pm 7.2$ & $14.7 \pm 9.1$ & $7.5 \pm 6.6$ & $13.3 \pm 8.2$ \\
Medication (on/off) & $0 / 24$ & $13 / 9$ & $0 / 29$ & $24 / 10$ \\
\hline
\end{tabular}

Abbreviations: female (f); male (m); post mortem interval (PMI). Values are presented as \pm standard deviation 